\title{
EDITORIAL
}

\section{Sleep apnoea: time to consider clock genes}

\author{
S. Andreas* and G. Eichele ${ }^{\#}$
}

M ost, and perhaps all, cells harbour a circadian clock. These clocks regulate hundreds of physiological processes, ranging from body temperature and sleep-wake cycle to serum cortisol and melatonin concentration. All clocks operate using a very similar molecular mechanism; they communicate with each other through a complex and still poorly understood neuroendocrine network [1] In the mammalian brain, for example, a key clock gene known as Bmal is turned on and off in a 24-h rhythm, not only in the suprachiasmatic nucleus of the hypothalamus (the seat of the master clock) but also in the hippocampus and neocortex [1]. It is therefore expected that many processes occurring in the neocortex are subject to clock control. The question, then, is how such control is achieved and which neuoendocrine regulators play a role. The study by BuRIOKA et al. [2] presented in the current issue of the European Respiratory Journal (ERJ) contributes significantly towards addressing this question.

The circadian rhythm of vigilance, blood pressure, sympathetic activity, corticoid biogenesis and metabolism is severely altered in patients with obstructive sleep apnoea (OSA). Given the previously sketched importance of clock genes in the genesis of circadian rhythm, investigation of circadian gene activation in OSA patients seems to hold promise. BURIOKA et al. [2] have quantified the expression of the clock gene Per1 mRNA in peripheral blood cells using real-time PCR analysis at different points over $24 \mathrm{~h}$. In contrast to the matched healthy controls, the eight OSA patients showed no daily variation of Per1 mRNA expression. Continuous positive airway pressure (CPAP) treatment for 3 months improved diurnal variation of Per1 mRNA expression and reduced interleukin (IL)-6, high-sensitivity Creactive protein and plasma noradrenaline in OSA patients. That these clear-cut results were found by investigating the rather small number of subjects (eight patients and eight controls) could be explained by the fact that patients with severe OSA (apnoea/hypopnoea index $54 \cdot \mathrm{h}^{-1}$ ) were carefully selected. Furthermore, the average percentage of CPAP use was fair $(75 \%)$ and details such as abstention from caffeine containing beverages were considered.

To unravel the underlying mechanism of clock gene dysfunction in OSA, BURIOKA et al. [2] performed further experiments in mice. The circadian clock in mice is regulated in a similar

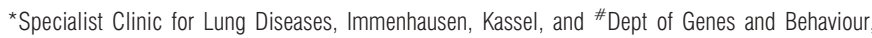
MPI Biophysical Chemistry, Göttingen, Germany.

STATEMENT OF INTEREST: None declared.

CORRESPONDENCE: S. Andreas, Lungenfachklinik Immenhausen, Pneumologische Lehrklinik der Universität Göttingen, Robert-Koch-Straße 3, D-34376 Immenhausen, Kreis Kassel, Germany. Fax: 49 5673501101. E-mail: sandreas@lungenfachklinik-immenhausen.de
}

manner to humans [1]. The continuous administration of noradrenaline by an osmotic mini pump for 6 days in three mice induced Per1 mRNA expression in the cerebral cortex and other tissues during the daytime when the mice were asleep [2]. Furthermore, in vitro experiments showed that mediators of inflammation such as IL-6 did not impact on Per1 mRNA expression [2]. In a previously published study, BuRIOKA et al. [3] found that a $\beta_{2}$-adrenoceptor agonist strongly induced human Per1 mRNA expression both in vitro and in vivo, thereby supporting the data presented in the current issue of the ERJ.

Ear2/Nr2f6 mutant mouse lack a sizable fraction of the locus coeruleus [4]. As this nucleus is the chief source of noradrenalin in the mammalian brain, the three-fold reduction in the concentration of noradrenalin in the brain of mutant mice comes as no surprise. An unexpected observation was that the circadian expression profile of Per1 typically seen in the neocortex of wild-type mice is abolished in the Ear2/Nr2f6 mutant animals [4]. No effect was seen on Per1 expression in the suprachiasmatic nucleus, suggesting that the master clock is fully operational. Behavioural studies showed that in Ear2/ Nr2f6 mutant mice, food anticipation was abnormal. It is thought that this particular behaviour is regulated by a circadian clock residing in the neocortex. The lesson to be learned from these studies with Ear2/Nr2f6 mutant mice is that noradrenalin is a regulator of circadian clocks residing in the cortex [4]. The previously mentioned experiments in mice performed by BURIOKA and co-workers $[2,3]$ further substantiate this idea.

With the advance of microneurography it became clear that the sympathoexitation that is present during sleep and wakefulness is striking and central to the pathogenesis of OSA [5-7]. While it is well established that the chronic sympathetic activation present in OSA negatively impacts on skeletal muscle function, arteriosclerosis, systemic inflammation and glucose metabolism, the underlying mechanism is less apparent [7-9].

The activation of the sympathetic nervous system on the morning following nocturnal restitution is a powerful zeitgeber (timekeeper) in humans [10]. Heart rate and blood pressure exhibit diurnal variations reflected by strong effects of the molecular circadian clock in myocardial and endothelial cells. For instance, the pyruvate dehydrogenase kinase 4 is four times more active during the day than it is at night [10]. In concert with the regulation of other enzymes, this favours carbohydrate oxidation during the daytime and fatty acid oxidation during the night-time. Thus, the circadian clock within the heart allows anticipation of diurnal variations in workload and substrate availability. Impairment of this molecular mechanism contributes to contractile dysfunction 
associated with dyslipidaemia [10]. Circadian clocks are altered in animal models of arterial hypertension and diabetes mellitus [10,11], and the clock null mouse is predisposed to obesity and metabolic syndrome [12]. The study by BURIOKA et al. [2] helps us understand how clock gene dysfunction due to sympathoexitation contributes to the association of OSA with cardiovascular disease. Additional studies will be necessary to better understand the effect of OSA on other clock genes, such as Per2, Cry and Bmal. The effects of OSA on diurnal variations in endothelial, myocardial, liver, inflammatory and fat cell function also need to be characterised.

Depression, impaired vigilance and impaired cognitive function are common in OSA patients and can be improved through CPAP treatment [13, 14]. Depression, particularly unipolar depression, is strongly linked to 24-h rhythm abnormalities, such as disruption of the sleep-wake cycle [14]. Both circadian rhythm of the central nervous system and the sleep-wake cycle are under the control of clock genes [15]. The molecular circadian clock is present in most cells of the central nervous system and regulates the timing of expression of $\geqslant 10 \%$ of the transcripts [14]. Interestingly, sleep disorders such as familial advanced sleep phase syndrome or delayed sleep phase syndrome show mutations in clock genes [15]. Therefore, clock gene dysfunction might help us to better understand how OSA is related to depression [13].

Analogous to OSA, patients with restrictive lung disease as well as chronic obstructive pulmonary disease (COPD) exhibit marked neurohumoral activation [16, 17]. It therefore seems worth considering clock gene dysfunction as a cause of systemic sequelae in COPD, such as skeletal muscle dysfunction, inflammation, cachexia and cardiovascular disease. COPD and asthma bronchiale are commonly treated with inhaled or even systemic $\beta_{2}$-agonists and glucocorticoids. Interestingly, both treatments strongly impact on clock gene expression [2].

In summary, there is ample evidence to suggest that circadian rhythm and clock genes play an essential role in cardiovascular, metabolic and pulmonary disease. Most importantly, obstructive sleep apnoea, a disease characterised by a treatable circadian rhythm disorder, might well be regarded as paradigmatic in providing new insights into human clock gene function.

\section{REFERENCES}

1 Albrecht U. Invited review: regulation of mammalian circadian clock genes. J Appl Physiol 2002; 92: 1348-1355.

2 Burioka N, Koyanagi S, Endo M, et al. Clock gene dysfunction in patients with obstructive sleep apnoea syndrome. Eur Respir J 2008; 32: 105-112.
3 Burioka N, Fukuoka Y, Takata M, et al. Circadian rhythms in the CNS and peripheral clock disorders: function of clock genes: influence of medication for bronchial asthma on circadian gene. J Pharmacol Sci 2007; 103: 144-149.

4 Warnecke M, Oster H, Revelli JP, Alvarez-Bolado G, Eichele G. Abnormal development of the locus coeruleus in Ear2(Nr2f6)-deficient mice impairs the functionality of the forebrain clock and affects nociception. Genes Dev 2005; 19: 614-625.

5 Hedner J, Darpo B, Ejnell H, Carlson J, Caidahl K. Reduction in sympathetic activity after long-term CPAP treatment in sleep apnoea: cardiovascular implications. Eur Respir J 1995; 8: 222-229.

6 Coy TV, Dimsdale JE, Ancoli-Israel S, Clausen J. Sleep apnoea and sympathetic nervous system activity: a review. J Sleep Res 1996; 5: 42-50.

7 McNicholas WT, Bonsigore MR. Sleep apnoea as an independent risk factor for cardiovascular disease: current evidence, basic mechanisms and research priorities. Eur Respir J 2007; 29: 156-178.

8 Yumino D, Bradley TD. Pathogenesis of atherosclerosis: is obstructive sleep apnea the new kid on the block? Am J Respir Crit Care Med 2007; 176: 634-635.

9 Lüthje L, Andreas S. Obstructive sleep apnea and coronary artery disease. Sleep Medicine Review 2008; 12: 19-31.

10 Young ME. The circadian clock within the heart: potential influence on myocardial gene expression, metabolism, and function. Am J Physiol Heart Circ Physiol 2006; 290: H1-H16.

11 Oishi K, Kasamatsu M, Ishida N. Gene- and tissuespecific alterations of circadian clock gene expression in streptozotocin-induced diabetic mice under restricted feeding. Biochem Biophys Res Commun 2004; 317: 330-334.

12 Turek FW, Joshu C, Kohsaka A, et al. Obesity and metabolic syndrome in circadian clock mutant mice. Science 2005; 308: 1043-1045.

13 Giles TL, Lasserson TJ, Smith BJ, White J, Wright J, Cates CJ. Continuous positive airways pressure for obstructive sleep apnoea in adults. Cochrane Database Syst Rev 2006; 3: CD001106.

14 Turek FW. From circadian rhythms to clock genes in depression. Int Clin Psychopharmacol 2007; 22: Suppl. 2, S1-S8.

15 Ebisawa T. Circadian rhythms in the CNS and peripheral clock disorders: human sleep disorders and clock genes. J Pharmacol Sci 2007; 103: 150-154.

16 Raupach T, Bahr F, Herrmann P, et al. Slow breathing reduces sympathoexcitation in chronic obstructive pulmonary disease. Eur Respir J 2008; [Epub ahead of print PMID: 18385175].

17 Andreas S, Anker SD, Scanlon PD, Somers VK. Neurohumoral activation as a link to systemic manifestation of chronic lung disease. Chest 2005; 128: 3618-3624. 